\title{
Energy Spectrum of Extremal Invariant States
}

\author{
Richard H. Herman and Daniel Kastler* \\ Department of Mathematics, Pennsylvania State University, University Park, Pennsylvania 16802, USA
}

\begin{abstract}
For an extremal invariant state $\omega$ of a weakly asymptotically abelian dynamical system we prove that the corresponding energy spectrum is either one-sided or the whole reals, or a periodic subgroup. The latter case implies abelianness of the algebra in the representation generated by $\omega$.
\end{abstract}

The purpose of this note is to extend to weakly clustering (i.e. extremal invariant) states of asymptotically abelian systems a spectral alternative useful e.g. in deriving the KMS condition from stability assumptions (Prop. 3 of [1], see also Theorem $6.1 \mathrm{of}[2]$ ). This result follows from the long known fact that the energy spectrum of clustering states is additive [3]. The generalization we present here is relevant to the description of e.g. crystal states in motion.

Assumptions. $\{\mathfrak{U}, R, \alpha\}$ is the triple of a $C^{*}$-algebra $\mathfrak{A}$ together with a strongly continuous one-parameter group $t \in \mathbb{R} \rightarrow \alpha_{t}$ of *-automorphisms of $\mathfrak{A}$. We consider an $\alpha$-invariant state $\omega$ of $\mathfrak{A}$ such that

(i) (asymptotic abelianness) for any two $A, B \in \mathfrak{A}$ the commutator $\left[\alpha_{t}(A), B\right]$ tends to zero in mean under all states of the normal folium of $\omega$ :

$$
\frac{1}{2 T} \int_{-T}^{+T}\left[C\left[\alpha_{t}(A), B\right] D\right) d t \underset{T \rightarrow \infty}{\rightarrow} 0, \quad A, B, C, D \in \mathfrak{A} .
$$

(ii) (weak clustering)

$$
\frac{1}{2 T} \int_{-T}^{+T} \omega\left(A \alpha_{t}(B)\right) d t \underset{T \rightarrow \infty}{\longrightarrow} \omega(A) \omega(B), \quad A, B \in \mathfrak{A} .
$$

Theorem. Assume the above situation. Let $(\pi, U)$ be the covariant representation of $\{\mathfrak{U}, R, \alpha\}$, on the Hilbert space $\mathscr{H}$ with cyclic invariant vector $\Omega$, generated by the state $\omega$ via the GNS construction. We have the following alternatives 
a) the spectrum $S$, of $U$, is one-sided (i.e. confined to the positive or negative reals)

b) $S$ covers the whole real line

c) $S=Z b$ for some $b \in \mathbb{R}$ and $\pi(\mathfrak{U})$ is commutative.

The proof uses the two following lemmas.

Lemma 1 (see [1], [2], [3]). Under the standing assumptions the spectrum $S$ of $U$ is a semi-group.

We recall that $p \in \mathbb{R}$ belongs to $S$ iff; to each compact neighborhood $V$ of $p$, there is an $A \in \mathfrak{U}$ with $\operatorname{Sp}^{\alpha}(A) \subset V$ and $\pi(A) \Omega \neq 0$. Now, with $p_{1}, p_{2} \in S$ and $V$ a neighborhood of $p=p_{1}+p_{2}$, pick neighborhoods $V_{i} \ni p_{i}, i=1,2$ such that $V_{1}$ $+V_{2} \subset V$; and $A_{i} \in \mathfrak{A}$ with $\operatorname{Sp}^{\alpha}\left(A_{i}\right) \subset V_{i}$, and $\pi\left(A_{i}\right) \Omega \neq 0, i=1,2$. One has then $\operatorname{Sp}^{\alpha}\left\{\alpha_{s}\left(A_{1}\right) A_{2}\right\} \subseteq V_{1}+V_{2} \subseteq V^{1}$.

Claim: $s$ can be choosen such that $\pi\left(\alpha_{s}\left(A_{i}\right) A_{2}\right) \Omega \neq 0$. Indeed the square of the norm of the latter equals

$$
\omega\left(A_{2}^{*} \alpha_{s}\left(A_{1}^{*} A_{1}\right) A_{2}\right)
$$

which, owing to (1) and (2), tends in means towards

$$
\omega\left(A_{1}^{*} A_{2}\right) \omega\left(A_{1}^{*} A_{1}\right)=\left\|\pi\left(A_{2}\right) \Omega\right\|^{2} \cdot\left\|\pi\left(A_{1}\right) \Omega\right\|^{2} .
$$

This last expression is non-vanishing by assumption and so the claim is proven, completing the lemma.

Lemma 2. Let $S$ be a closed subset of the real line containing $\{0\}$. Suppose that $S$ is a semi-group under the usual addition. If there are points in $S$ on both sides of the origin then either $S=\mathbb{R}$ or $S=Z b$ for some $b \geqq 0$.

We proceed under the assumption $S \neq\{0\}$.

Suppose 0 is a accumulation point of $S$. Suppose, e.g. that $S \ni a_{i} \geqq 0 \underset{i=\infty}{\longrightarrow} 0$. Let $p \geqq 0$ and $\varepsilon>0$. There is an $a_{k}<\varepsilon$ and so there is an integer $n$ such that

$$
p=n a_{k}+r \text { with } r<\varepsilon .
$$

By the assumed semi-group property, $n a_{k} \in S$ and, since $S$ is also closed, $p \in S$. Thus all positive numbers belong to $S$ and since one negative number does, $S=\mathbb{R}$.

Suppose 0 is not a limit point of $S$. Let $-a, b ; a, b>0$ be the elements of $S$ closest to 0 from the negative and positive side respectively. We claim that $a=b$. This is clear by the semi-group property for if $a>b$ then $-a+b$ belongs to $S$ and $-a<-a$ $+b<0$. Now suppose $x$ is in $S, x$ not in $Z b$. Then,

$$
x=m b+r, \quad 0<r<b .
$$

However $-m b$ belongs to $S$ and thus so does $r$, contradicting the choice of $b$.

The proof of the lemma is now complete.

1 see e.g. [4] 
Proof of the Theorem. Suppose $S$ is neither one-sided nor the whole reals. By Lemma $2, S=Z b=\{n b ; n=0, \pm 1, \pm 2 \ldots\}$ for some $b \in \mathbb{R}$ which we first assume not to vanish. In that case, one immediately sees using Stone's theorem that $U$ is periodic i.e.

$$
U\left(t+\frac{2 \pi}{b}\right)=U(t), \quad t \in R .
$$

We next show that the action of $t \rightarrow \operatorname{Ad} U(t)$ on the weak closure $\mathscr{M}=\pi(\mathfrak{U})^{\prime \prime}$ of $\pi(\mathfrak{U})$ is ergodic (i.e. has only the scalars as fixed points). First any $A \in \mathfrak{U}$ such that $\alpha_{t}(A)=A$, $t \in R$, is such that $\pi(A)=\omega(A) I$. Indeed, by (i) and (ii), for $B_{1}, B_{2} \in \mathfrak{U}$

$$
\left(\pi\left(B_{1}\right) \Omega\left|\pi\left(\alpha_{t}(A)\right)\right| \pi\left(B_{2}\right) \Omega\right)=\omega\left(B_{1}^{*} \alpha_{t}(A) B_{2}\right)
$$

tends in mean toward

$$
\omega\left(B_{1}^{*} B_{2}\right) \omega(A)=\omega(A)\left(\pi\left(B_{1}\right) \Omega \mid \pi\left(B_{2}\right) \Omega\right)
$$

whence our claim by the cyclicity of $\Omega$. Now, by the periodicity stated above we really have a representation of the compact group $\mathbb{R} / Z \frac{2 \pi}{b}$. Therefore the "average" $\varepsilon$ given by

$$
\varepsilon(X)=\frac{b}{2 \pi} \int_{0}^{\frac{2 \pi}{b}} U(t) X U(t)^{*} d t, \quad X \in \mathscr{M},
$$

is manifestly a normal (see e.g. [5]) expectation onto the fixed points of $\mathscr{M}$ under $t \rightarrow \operatorname{Ad} U(t)$. Thus the fixed points in $\mathscr{M}$, are the strong closure of the fixed points in $\pi(\mathfrak{A})$, and so are just scalars.

We now appeal to a Theorem of Størmer (Theorem 3.5 of [6]) according to which a von Neumann algebra $\mathscr{M}$, with an ergodic action of the real line whose spectrum is not the whole real line, is abelian. For completeness we sketch the proof of this fact: "each $X \in \mathscr{M}$ has a strongly converging Fourier decomposition

$$
X=\sum_{n \in Z} \varepsilon_{n}(X), \quad \varepsilon_{n}(X)=\frac{b}{2 \pi} \int_{0}^{\frac{2 \pi}{b}} e^{-i n t} U(t) X U(t)^{*} d t .
$$

By ergodicity the $\varepsilon_{n}(X), X \in \mathscr{M}$ are all multiples of a fixed unitary $U_{n} \in \mathscr{M}$, for each $n \in Z$. Hence $U_{n}=\lambda_{n} U_{1}^{n}, n \in Z, \lambda_{n} \in \mathbb{C}$. Thus all the $U_{n}$ commute and so $\mathscr{M}$ is thus commutative by $(4)^{\prime \prime}$.

The case where $b=0$ yields, by the above reasoning the fact that $\pi(\mathfrak{U})$ reduces to the scalars, and thus is trivially abelian.

Remark 1. The alternative c) in the Theorem is excluded in the two following cases

1) The algebra $\mathfrak{A}$ is (non-abelian and) simple (since $\pi$ is then faithful)

2) The state $\omega$ enjoys the stronger clustering property

$$
\frac{1}{2 T} \int_{-T}^{+T}\left|\omega\left(A \alpha_{t}(B)\right)-\omega(A) \omega(B)\right| d t \underset{T=\infty}{\longrightarrow} 0, \quad A, B \in \mathfrak{U}
$$

(the discrete spectrum of $U$ then reduces to $0-\mathrm{cf}$. [7]). 
Remark 2. The above argument allows us to treat the following situation of physical interest: in addition to the above assumption (i), (ii) relative to the one-parameter group $t \rightarrow \alpha_{t}$ we assume that.

(iii) there is another one parameter group $x \in \mathbb{R} \rightarrow \beta_{x}$ of automorphisms of $\mathfrak{A}$, leaving $\omega$ invariant, commuting with $\alpha\left(\alpha_{t} \beta_{x}=\beta_{x} \alpha_{t}, t, x \in \mathbb{R}\right)$ and asymptotically abelian in the same way as $\alpha$.

(iv) the algebra $\mathfrak{A}$ has a trivial center (e.g. is simple).

The conclusions of the theorem then hold for the spectrum of the unitary representation of $\beta$ in the GNS construction by $\omega$ : indeed the proof of Lemma 1 can be extended to this case (since the $\alpha_{t}$ leave the spectral subspace of $\beta$ invariant); and the fixed points of $\mathfrak{U}$ under $\beta$ reduce to the scalars by (iv) and the asymptotic abelianness of $\beta$.

Acknowledgements. The authors gratefully acknowledge the hospitality of Professor Ludwig Streit at ZIF-Universität Bielefeld and of Professor Richard Kadison of the Mathematics Department at the University of Pennsylvania, which made their collaboration possible. The first named author is grateful for the support of the Humboldt-Stiftung and the US National Science Foundation.

\section{References}

1. Haag, R., Kastler,D., Trych-Pohlmeyer,E.: Stability and equilibrium states. Commun. math. Phys. 38, 173 (1974)

2. Kastler,D.: Equilibrium states of matter and operator algebras. In: Proceedings of the March 1975 Rome Conference "Algebre $C^{*}$ e loro applicazioni in Fisica teorica". Symp. Math. 20, 49 (1976)

3. Wightman, A.: Some results of the structure of relativistic quantum field theory. In: Proceedings of the international congress of mathematicians. Uppsala: Almqvist \& Wiksell 1963

4. Arveson, W.: On groups of automorphisms of operator algebras. J. Funct. Anal. 15, 217 (1974)

5. Herman, R.: Invariant states. Trans. A.M.S. 158, 503 (1971)

6. Størmer,E.: Spectra of ergodic transformations. J. Funct. Anal. 15, 202 (1974)

7. Doplicher, S., Kastler,D. : Ergodic states in a non-commutative ergodic theory. Commun. math. Phys. 1, (1968)

8. Kastler,D., Robinson,D.W.: Invariant states in statistical mechanics. Commun. math. Phys. 3, 151 (1966)

Communicated by J. L. Lebowitz

Received April 11, 1977

\section{Note Added in Proof}

Derek Robinson has reminded one of us (D. K.) that the abelianess of $\pi(\mathfrak{H})$ in the case c) of our Theorem also follows from [8] Lemma 6 (see Lemma 4 of [7] for the case of a non commutative group). 